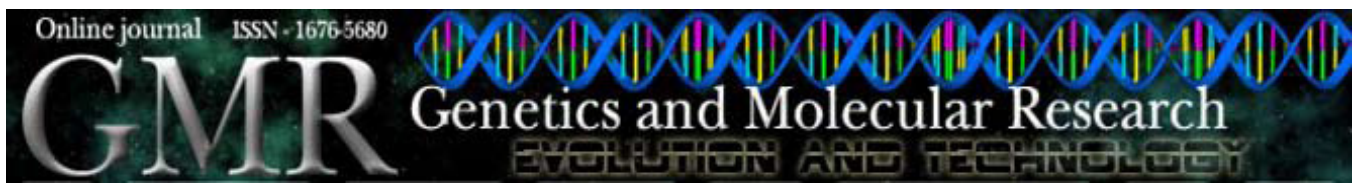

\title{
GSTP1 Ile105Val polymorphism in astrocytomas and glioblastomas
}

\author{
A.C. Custódio ${ }^{1}$, L.O. Almeida ${ }^{1}$, G.R. Pinto ${ }^{1,3}$, M.J. Santos ${ }^{2}$, \\ J.R.W. Almeida ${ }^{2}$, C.A. Clara ${ }^{2}$, J.A. Rey ${ }^{4}$ and C. Casartelli ${ }^{1}$ \\ ${ }^{1}$ Departamento de Genética, Laboratório de Oncogenética, \\ Faculdade de Medicina de Ribeirão Preto, Universidade de São Paulo, \\ Ribeirão Preto, SP, Brasil \\ ${ }^{2}$ Fundação Pio XII, Hospital de Câncer de Barretos, Barretos, SP, Brasil \\ ${ }^{3}$ Laboratório de Genética Humana e Biologia Molecular, \\ Universidade Federal do Piauí, Parnaíba, PI, Brasil \\ ${ }^{4}$ Departamento de Cirurgia Experimental, Laboratório de Oncogenética \\ Molecular, Hospital Universitário "La Paz", Madrid, Espanha \\ Corresponding author: A.C. Custódio \\ E-mail: alinecadurin@yahoo.com.br
}

Genet. Mol. Res. 9 (4): 2328-2334 (2010)

Received August 12, 2010

Accepted September 11, 2010

Published November 30, 2010

DOI 10.4238/vol9-4gmr971

\begin{abstract}
Glutathione S-transferases (GSTs) constitute a superfamily of ubiquitous multifunctional enzymes that are involved in the cellular detoxification of a large number of endogenous and exogenous chemical agents that have electrophilic functional groups. People who have deficiencies in this family of genes are at increased risk of developing some types of tumors. We examined GSTP1 Ile105Val polymorphism using PCR-RFLP in 80 astrocytoma and glioblastoma samples. Patients who had the Val allele of the GSTP1 Ile105Val polymorphism had an increased risk of tumor development (odds ratio $=8.60 ; 95 \%$ confidence interval $=4.74-17.87 ; \mathrm{P}<0.001)$. Overall survival of patients did not differ significantly. We suggest that GSTP1 Ile105Val polymorphisms are involved in susceptibility to developing astrocytomas and glioblastomas.
\end{abstract}

Key words: Polymorphism; GSTP1; Astrocytoma; Glioblastoma 


\section{INTRODUCTION}

Tumors of the central nervous system (CNS) represent approximately $2 \%$ of all cancers, with an estimated 4.2 to 5.4 per 100.000 individuals per year (Ohgaki and Kleihues, 2005). Although the incidence of CNS tumors is small compared to other cancers, these are among the most serious human malignancies, since they affect the coordination and integration of all body activities. Moreover, as each region of the brain has a vital function, the therapy used in other cancers (total surgical removal of the organ or tumor with a generous margin of normal tissue) cannot be applied to cure patients with brain tumors (Louis et al., 2002; Ohgaki and Kleihues, 2005). Gliomas are the most common tumors of the CNS. Despite the remarkable progress in the characterization of the molecular pathogenesis of gliomas, these tumors remain incurable and, in most cases, refractory to treatment due to their molecular heterogeneity (Kleihues et al., 2002).

Astrocytomas account for the large majority of gliomas, making up $70 \%$ of the total, and can be divided into: pilocytic astrocytomas (grade I), including low-grade astrocytomas (grade II), anaplastic (grade III), and glioblastoma (grade IV) (Kleihues et al., 2002). The importance of the grading scheme of malignancy based on histopathology is indicated by the patient's survival. Patients with low-grade astrocytomas (grade II) have a median survival of about seven years, and patients with anaplastic astrocytomas (grade III) have a mean survival of half that time (McCormack et al., 1992), while patients with glioblastoma have an average of 9 to 11 months (Simpson et al., 1993). Unlike astrocytomas, the progression of grade II and III tumors for most malignant tumors is well documented (Ino et al., 2001; Collins, 2004; Hartmann et al., 2004; Ichimura et al., 2004; Ohgaki, 2005). Ng and Lam (1998) suggested the division of glioblastomas into two clinical and molecular distinct entities: primary glioblastomas or new ones, which occur in elderly patients and are clinically very aggressive, and secondary glioblastomas, which develop from preexisting low-degree astrocytomas and have a more prolonged clinical course.

Several studies have examined the relationship of this polymorphism with the formation of various types of tumors such as lung, head and neck, testicular, and breast (Ezer et al., 2002). The presence of this polymorphism is involved in reducing the activity of GSTP1 in $78 \%$ of children with asthma in Taiwan (Kabesch et al., 2004). Ezer et al. (2002) conducted a study on brain tumors and concluded that the presence of the Ile105Val polymorphism may lead to the development of pediatric astrocytomas and their progression to malignant grades. The GSTP1 gene is located on chromosome 11 in the 11q13 region and possesses nine exons (White et al., 2008). This gene accounts for about $90 \%$ of the enzymatic activity of the glutathione S-transferase (GST) family. The activity of GSTP1 enzyme can be altered by the presence of the polymorphism Ile105Val. This polymorphism is located in exon 5 of the gene (rs947894) (McLeish and Turner, 2007).

In the present research, a case-control study was conducted to examine the genotype distribution of Ile105Val single-nucleotide polymorphism (SNP) and to search for an association between astrocytomas and glioblastomas and GSTP1 SNP, using the polymerase chain reaction-restriction fragment length polymorphism (PCR-RFLP) approach.

\section{MATERIAL AND METHODS}

\section{Study population}

Eighty gliomas were analyzed; they had been surgically resected from previously un- 
treated patients under the care of the Neurosurgery Department of Fundação Pio XII, Cancer Hospital of Barretos (Barretos, SP, Brazil). The samples, classified according to WHO criteria, were: 43 astrocytomas and 37 glioblastomas. The clinical outcome, including length of survival, was obtained from patient records. For SNP studies, blood samples were collected from 100 healthy individuals to serve as controls. Because of the highly heterogeneous ethnic composition of the Brazilian population, the individuals of the control group were selected from the general population of São Paulo State, with no family history of cancer in first-degree relatives.

\section{DNA extraction and primer construction}

DNA extraction was performed using proteinase $\mathrm{K}$ and phenol-chloroform according to routine molecular biology protocols. Primers were constructed using the Gene Runner 3.05 program (Hasting Software, Inc.) from the gene sequence of the GSTP1 Ile105Val polymorphism, obtained from the dbSNP of NCBI (accession No. rs 947894). Table 1 shows the primers and PCR product sizes.

Table 1. Polymerase chain reaction (PCR) primers.
\begin{tabular}{lllcc}
\hline SNP & Primer & Sequence & Length (bp) & PCR product (bp) \\
\hline GSTP1 Ile105Val & Ile105Val-F & CCT GCT CCC CTC CAC CCA AC & 20 & 180 \\
& Ile105Val R & GCC CCT TTC TTT GTT CAG C & 19 & \\
\hline
\end{tabular}

$\mathrm{SNP}=$ single-nucleotide polymorphism.

PCR was carried out in a final volume of $25 \mu \mathrm{L}$ containing 50 ng genomic DNA template, 1X PCR buffer with $2 \mathrm{mM} \mathrm{MgCl}, 0.4 \mu \mathrm{M}$ of each primer (Invitrogen), $50 \mu \mathrm{M}$ dNTPs (Amersham Biosciences) and 0.5 U DNA polymerase (Biotools). The PCR cycling conditions were: $94^{\circ} \mathrm{C}$ for $5 \mathrm{~min}$, followed by 35 denaturation cycles of $30 \mathrm{~s}$ at $94^{\circ} \mathrm{C}, 30 \mathrm{~s}$ of annealing at $60^{\circ} \mathrm{C}$, and $30 \mathrm{~s}$ of extension at $72^{\circ} \mathrm{C}$, and a final elongation cycle at $72^{\circ} \mathrm{C}$ for $5 \mathrm{~min}$. For RFLP, the PCR products were digested by $B S M A\left(10 \mathrm{U}\right.$ at $55^{\circ} \mathrm{C}$ for $\left.5 \mathrm{~h}-\mathrm{Ile} 105 \mathrm{Val}\right) . B S M A$ recognizes a restriction site at Val105 (GTCTCN/NNNN) and generates two fragments of different sizes (115 and $65 \mathrm{bp}$ ), while Ile105 allele generates only one fragment of $180 \mathrm{bp}$. DNA fragments were electrophoresed through a 10\% acrylamide:bisacrylamide gel (19:1) and stained with silver nitrate.

\section{Statistical analysis}

PCR-RFLP - The independence of alleles (Hardy-Weinberg equilibrium) was ensured using the chi-square test. The distribution of genotype and allele frequencies among patients and controls was compared using chi-square and Fisher exact tests. Overall survival curves were obtained using the Kaplan-Meier method and compared with a log-rank test. Odds ratio (OR) and 95\% confidence intervals (CI) were calculated using a logistic regression model. Statistical significance was set at $\mathrm{P}<0.05$. Statistical analyses were performed with GraphPad InStat 4.0 and GraphPad Prism 5.0 softwares (GraphPad Software, Inc.). 


\section{RESULTS}

\section{Analysis of tumor and control populations according to the GSTP1 Ile105Val}

Eighty patients and 100 control subjects were included in this study. The patient sample comprised 28 females and 52 males $(\mathrm{M} / \mathrm{F}$ ratio $=0.65)$, and the control sample consisted of 63 males and 37 females $(\mathrm{M} / \mathrm{F}$ ratio $=1.7)$. The average age of the patient group was 45 years (range $=1-75$ ) and of the control group, 45 years (range $=18-72$ ). Genotype frequencies in controls and patients were in Hardy-Weinberg equilibrium. Allele and genotype frequencies of GSTP1 Ile105Val in controls and patients are shown in Table 2. The frequencies of Ile/ Ile and Ile/Val among controls were 87 and 13\%, while in patients the frequencies Ile/Ile, Ile/ $\mathrm{Val}$ and $\mathrm{Val} / \mathrm{Val}$ were $43.8,53.8$ and $2.5 \%$, respectively $(\mathrm{P}<0.0001)$; Val105 allele frequency was statistically significant between cases and controls $(0.29$ and 0.06 , respectively, $\mathrm{P}<0.001)$.

\begin{tabular}{|c|c|c|c|c|}
\hline SNP & Genotype & Case group & Control group & $\mathrm{P}$ \\
\hline \multirow[t]{4}{*}{ GSTP1 Ile105Val } & Ile/Ile & $35(43.8 \%)$ & $87(87 \%)$ & $<0.001$ \\
\hline & Ile/Val & $43(53.8 \%)$ & $13(13 \%)$ & \\
\hline & $\mathrm{Val} / \mathrm{Val}$ & $2(2.5 \%)$ & $0(0)$ & \\
\hline & Val105 allele frequency & 0.29 & 0.06 & $<0.001$ \\
\hline
\end{tabular}

Data are reported as numbers with percent in parentheses. $\mathrm{SNP}=$ single-nucleotide polymorphism.

Logistic regression analysis for the investigation of polymorphism association with risk of astrocytomas and glioblastomas is presented in Table 3. Compared to Ile/Ile, the most common genotype of the polymorphism GSTP1 Ile105Val in the study population, the genotypes with presence of allele Val revealed an increased risk of tumor development $(\mathrm{OR}=8.60 ; 95 \% \mathrm{CI}=4.14$ $17.87 ; \mathrm{P}<0.001)$.

\begin{tabular}{llccr}
\multicolumn{5}{l}{ Table 3. Association of GSTP1 Ile105Val single-nucleotide polymorphisms (SNP) with risk of cancer. } \\
\hline SNP & Genotype & Case/Control & OR (95\%CI) & P \\
\hline GSTP1 Ile105Val & Ile/Ile & $35 / 87$ & Ref. & - \\
& Ile/Val & $43 / 13$ & $8.22(3.94-17.13)$ & $<0.001$ \\
& Val/Val & $2 / 0$ & - & 0.029 \\
& Ile/Val or Val/Val & $45 / 13$ & $8.60(4.14-17.87)$ & $<0.001$ \\
\hline
\end{tabular}

$\mathrm{OR}=$ odds ratio $; 95 \% \mathrm{CI}=$ confidence interval at $95 \%$.

Comparison of overall survival of patients according to GSTP1 Ile105Val genotype did not show significant differences $(\mathrm{P}=0.82)$. In the GSTP1 Ile105Val genotype, the median survival of patients with Ile/Val and Val/Val was 63 weeks (Figure 1).

\section{DISCUSSION}

The concept of neoplastic transformation links together two types of genetic background of cancer with the same final results. Besides entirely somatic cell gene deregulation, a genetically determined susceptibility is taken into account. The predisposition to cancer results from the inheritance of altered alleles of genes, which are usually of the tumor suppressor type. GSTs constitute a superfamily of ubiquitous multifunctional enzymes that are 


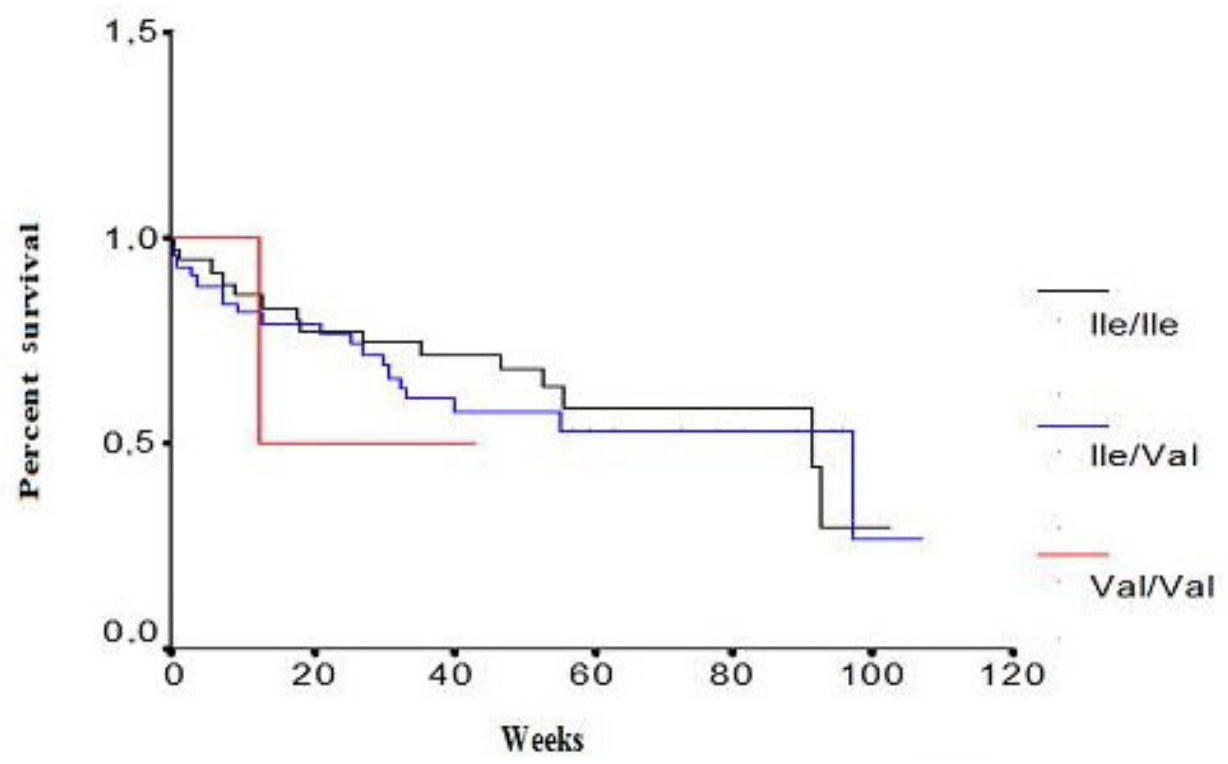

Figure 1. Overall survival in patients according to GSTP1 Ile105Val single-nucleotide polymorphism. P=0.82.

involved in the cellular detoxification of a large number of endogenous and exogenous chemical agents that have electrophilic functional groups (Ryberg et al., 1997). The GSTP1 gene encodes the pi class of enzymes and this gene has been extensively examined in association with risk of cancer (White et al., 2008). The influence of GSTP1 Ile105Val polymorphism on cancer with inconsistent results has been reported from different parts of the world (Syamala et al., 2008).

In this study, we determined the relationship between GSTP1 Ile105Val SNPs and susceptibility to cancer and patient survival in 80 astrocytomas and glioblastomas. Several studies have examined the relationship between this polymorphism and the development of various types of tumors such as lung, head and neck, testicular, and breast (Ezer et al., 2002). The presence of this polymorphism was shown to be involved in reducing the activity of GSTP1 in 78\% of children with asthma in Taiwan (Kabesch et al., 2004). In another study, Millikan et al. (2000) reported that the presence of this polymorphism in families leads to predisposition to the growth of tumors in the breast.

The present case-control study showed that the Val105 was more frequent in the cancer population than in non-cancer population $(0.29$ and 0.06 , respectively; $\mathrm{P}<0.001)$, and that the presence of this genotype may increase the risk of developing astrocytomas and glioblastomas $(\mathrm{OR}=8.60 ; 95 \% \mathrm{CI}=4.14-17.87 ; \mathrm{P}<0.001)$. Ezer et al. $(2002)$ conducted a study in brain tumors and concluded that the presence of the Ile105Val polymorphism may lead to the development of pediatric astrocytomas and their development to malignant grades. Singh et al. (2008) reported the presence of the Ile105Val polymorphism in individuals with head and neck tumors, suggesting that this polymorphism could lead to tumor development when compared to the control population.

Reszka et al. (2007) analyzed the influence of this polymorphism on the risk of developing lung cancer. They observed a large accumulation of oxidants in the blood of 
individuals who had the variant Val105 and also found higher frequencies of the Val/Val genotype among patients with lung cancer than in the control group. Saxena et al. (2009) conducted a case-control study in individuals of the population of northern India in an attempt to assess the relationship between polymorphism Ile105Val of GSTP1 and risk of breast cancer. They observed a 2.6 times greater chance of developing this type of cancer in individuals with genotype Val/Val. Kadouri et al. (2008) analyzed the influence of the Ile105Val polymorphism in patients with breast cancer who also had mutations in the BRCA gene and observed a 3.2 times higher risk among carriers of the Val105 allele, suggesting that the presence of the Val105 variant may be associated with the development of breast cancer.

Ye et al. (2006) conducted a study involving 23,000 individuals with lung cancer and 30,000 controls and saw no association between polymorphism GSTP1 Ile/Val and the risk of lung cancer. Wideroff et al. (2007) also found no relationship between the development of gastric adenocarcinoma and the presence of polymorphic variant Val105 of gene GSTP1. De Roos et al. (2003) analyzed 422 patients with gliomas, 172 patients with meningiomas and 79 schwannomas and observed that the presence of the Val/Val genotype was associated with increased incidence of gliomas and schwannomas, and that risk increased according to the number of Val alleles (1.3-fold for Ile/Val and 2.1 times for Val/ Val). Schwartzbaum et al. (2007) analyzed 329 cases of meningiomas and 546 gliomas and found no association between the risk of tumors of the nervous system and the presence of GSTP1 polymorphism Ile105Val.

De Roos et al. (2003) analyzed samples of gliomas, meningiomas and neuromas and concluded that the presence of the Ile105Val polymorphism is associated with the risk of such patients developing gliomas. Okcu et al. (2004) analyzed the Ile105Val polymorphism in 278 patients with malignant gliomas and also noted that its presence leads to the development of tumors.

In summary, our study provides evidence that GSTP1 Ile105Val may contribute to the etiology of human astrocytomas and glioblastomas, since allele Val105 was found more frequently in patients than controls, but there was no association between the genotypes and the patients' survival.

\section{ACKNOWLEDGMENTS}

We are grateful to the patients who took part in this investigation. We thank V.M. de Oliveira and M.R. Penha for the technical support provided in this study. Research supported by Conselho Nacional de Desenvolvimento Científico e Tecnológico (CNPq), Fundação de Amparo à Pesquisa do Estado de São Paulo (FAPESP) and Fundação de Apoio ao Ensino, Pesquisa e Assistência do Hospital das Clínicas da Faculdade de Medicina de Ribeirão Preto (FAEPA).

\section{REFERENCES}

Collins VP (2004). Brain tumours: classification and genes. J. Neurol. Neurosurg. Psychiatry 75 (Suppl 2): ii2-ii11.

De Roos AJ, Rothman N, Inskip PD, Linet MS, et al. (2003). Genetic polymorphisms in GSTM1, -P1, -T1, and CYP2E1 and the risk of adult brain tumors. Cancer Epidemiol. Biomarkers Prev. 12: 14-22.

Ezer R, Alonso M, Pereira E, Kim M, et al. (2002). Identification of glutathione S-transferase (GST) polymorphisms in 
brain tumors and association with susceptibility to pediatric astrocytomas. J. Neurooncol. 59: 123-134.

Hartmann C, Mueller W and von Deimling A (2004). Pathology and molecular genetics of oligodendroglial tumors. $J$. Mol. Med. 82: 638-655.

Ichimura K, Ohgaki H, Kleihues P and Collins VP (2004). Molecular pathogenesis of astrocytic tumours. J. Neurooncol. 70: $137-160$.

Ino Y, Betensky RA, Zlatescu MC, Sasaki H, et al. (2001). Molecular subtypes of anaplastic oligodendroglioma: implications for patient management at diagnosis. Clin. Cancer Res. 7: 839-845.

Kabesch M, Hoefler C, Carr D, Leupold W, et al. (2004). Glutathione S transferase deficiency and passive smoking increase childhood asthma. Thorax 59: 569-573.

Kadouri L, Kote-Jarai Z, Hubert A, Baras M, et al. (2008). Glutathione-S-transferase M1, T1 and P1 polymorphisms, and breast cancer risk, in BRCA1/2 mutation carriers. Br. J. Cancer 98: 2006-2010.

Kleihues P, Louis DN, Scheithauer BW, Rorke LB, et al. (2002). The WHO classification of tumors of the nervous system. J. Neuropathol. Exp. Neurol. 61: 215-225.

Louis DN, Pomeroy SL and Cairncross JG (2002). Focus on central nervous system neoplasia. Cancer Cell 1: 125-128.

McCormack BM, Miller DC, Budzilovich GN, Voorhees GJ, et al. (1992). Treatment and survival of low-grade astrocytoma in adults - 1977-1988. Neurosurgery 31: 636-642.

McLeish S and Turner SW (2007). Gene-environment interactions in asthma. Arch. Dis. Child 92: 1032-1035.

Millikan R, Pittman G, Tse CK, Savitz DA, et al. (2000). Glutathione S-transferases M1, T1, and P1 and breast cancer. Cancer Epidemiol. Biomarkers Prev. 9: 567-573.

Ng HK and Lam PY (1998). The molecular genetics of central nervous system tumors. Pathology 30: 196-202.

Ohgaki H (2005). Genetic pathways to glioblastomas. Neuropathology 25: 1-7.

Ohgaki H and Kleihues P (2005). Epidemiology and etiology of gliomas. Acta Neuropathol. 109: 93-108.

Okcu MF, Selvan M, Wang LE, Stout L, et al. (2004). Glutathione S-transferase polymorphisms and survival in primary malignant glioma. Clin. Cancer Res. 10: 2618-2625.

Reszka E, Wasowicz W and Gromadzinska J (2007). Antioxidant defense markers modulated by glutathione S-transferase genetic polymorphism: results of lung cancer case-control study. Genes Nutr. 2: 287-294.

Ryberg D, Skaug V, Hewer A, Phillips DH, et al. (1997). Genotypes of glutathione transferase M1 and P1 and their significance for lung DNA adduct levels and cancer risk. Carcinogenesis 18: 1285-1289.

Saxena A, Dhillon VS, Raish M, Asim M, et al. (2009). Detection and relevance of germline genetic polymorphisms in glutathione S-transferases (GSTs) in breast cancer patients from northern Indian population. Breast Cancer Res. Treat. 115: 537-543.

Schwartzbaum JA, Ahlbom A, Lonn S, Warholm M, et al. (2007). An international case-control study of glutathione transferase and functionally related polymorphisms and risk of primary adult brain tumors. Cancer Epidemiol. Biomarkers Prev. 16: 559-565.

Simpson JR, Horton J, Scott C, Curran WJ, et al. (1993). Influence of location and extent of surgical resection on survival of patients with glioblastoma multiforme: results of three consecutive Radiation Therapy Oncology Group (RTOG) clinical trials. Int. J. Radiat. Oncol. Biol. Phys. 26: 239-244.

Singh M, Shah PP, Singh AP, Ruwali M, et al. (2008). Association of genetic polymorphisms in glutathione S-transferases and susceptibility to head and neck cancer. Mutat. Res. 638: 184-194.

Syamala VS, Sreeja L, Syamala V, Raveendran PB, et al. (2008). Influence of germline polymorphisms of GSTT1, GSTM1, and GSTP1 in familial versus sporadic breast cancer susceptibility and survival. Fam. Cancer 7: 213-220.

White DL, Li D, Nurgalieva Z and El-Serag HB (2008). Genetic variants of glutathione S-transferase as possible risk factors for hepatocellular carcinoma: a HuGE systematic review and meta-analysis. Am. J. Epidemiol. 167: 377-389.

Wideroff L, Vaughan TL, Farin FM, Gammon MD, et al. (2007). GST, NAT1, CYP1A1 polymorphisms and risk of esophageal and gastric adenocarcinomas. Cancer Detect. Prev. 31: 233-236.

Ye Z, Song H, Higgins JP, Pharoah P, et al. (2006). Five glutathione s-transferase gene variants in 23,452 cases of lung cancer and 30,397 controls: meta-analysis of 130 studies. PLoS Med. 3: e91. 